\title{
En quoi la diversité des stratégies pédagogiques participe-t-elle à la motivation à apprendre des étudiants? Etude d'un cas particulier
}

Émilie Tremblay-Wragg, Carole Raby et Louise Ménard

\section{(2) OpenEdition Journals}

Édition électronique

URL : http://journals.openedition.org/ripes/1288

DOI : 10.4000/ripes. 1288

ISSN : 2076-8427

Éditeur

Association internationale de pédagogie universitaire

Référence électronique

Émilie Tremblay-Wragg, Carole Raby et Louise Ménard, « En quoi la diversité des stratégies pédagogiques participe-t-elle à la motivation à apprendre des étudiants ? Etude d'un cas particulier », Revue internationale de pédagogie de l'enseignement supérieur [En ligne], 34(1) | 2018, mis en ligne le 26 mars 2018, consulté le 10 décembre 2020. URL : http://journals.openedition.org/ripes/1288 ; DOI :

https://doi.org/10.4000/ripes. 1288

Ce document a été généré automatiquement le 10 décembre 2020.

Article L.111-1 du Code de la propriété intellectuelle. 


\title{
En quoi la diversité des stratégies pédagogiques participe-t-elle à la motivation à apprendre des étudiants? Etude d'un cas particulier
}

\author{
Émilie Tremblay-Wragg, Carole Raby et Louise Ménard
}

\section{Introduction}

1 Depuis l'avènement de l'enseignement universitaire de masse, la population étudiante s'est considérablement modifiée (Altet, 2009). Cette situation a nécessité l'adaptation des enseignants universitaires à une population étudiante hétérogène (Loiola et Romainville, 2008) et une remise en question des stratégies pédagogiques traditionnelles. Selon Prégent, Bernard et Kozanitis (2009), les étudiants font preuve d'une attitude positive envers les études. Ils souhaitent apprendre et désirent relever des défis importants dans le cadre de leur cheminement académique. Pourtant, le taux de réussite des étudiants au baccalauréat (premier cycle universitaire au Québec) est préoccupant (Gouvernement du Québec, 2014). Pour tenter d'agir sur le taux d'abandon élevé des études universitaires qui engendre des conséquences à la fois sur l'étudiant, sur la société et sur les universités (Sauvé, Debeurme, Wright, Fournier et Fontaine, 2007), il est important de comprendre ce qui affecte la réussite et la persévérance aux études postsecondaires dans le but d'offrir aux étudiants de bonnes conditions d'enseignement et d'apprentissage.

2 Plusieurs auteurs, dont Pintrich et De Groot (1990), soutiennent que l'utilisation de stratégies pédagogiques diversifiées est motivante pour les étudiants et a un effet positif sur leur réussite et leur persévérance scolaires. Comme plusieurs autres chercheurs intéressés par la motivation dans une perspective sociocognitive (Eccles et 
Wigfield, 2002 ; Pintrich et Schrauben, 1992), Viau (2009) reconnaît le rôle des activités pédagogiques et des pratiques évaluatives dans la motivation des étudiants. Les choix pédagogiques d'un professeur peuvent être déterminants sur l'engagement des étudiants dans un cours universitaire (Viau, 2014). Un enseignant qui priorise des stratégies pédagogiques favorisant l'engagement cognitif des étudiants contribue au choix des étudiants à s'engager cognitivement et à persévérer dans la tâche. L'utilisation de plusieurs stratégies en lien avec la motivation des étudiants apparait toutefois peu documentée scientifiquement. La description des stratégies employées par le professeur d'université présenté dans cet article ${ }^{1}$ contribue à cerner les facteurs qui peuvent susciter la motivation à apprendre des étudiants universitaires et suggère des modalités à mettre en place dans un contexte d'enseignement axé sur l'apprentissage afin de favoriser la réussite et la persévérance des étudiants. Le portrait de l'utilisation des stratégies pédagogiques à l'université ainsi que sa participation à la motivation des étudiants est d'abord dressé. Ensuite, la description du cas ainsi que la méthode utilisée pour répondre aux questions de recherche sont détaillées. Enfin, les résultats sont présentés et discutés.

\section{Importance des stratégies pédagogiques utilisées à l'université}

3 Par son enseignement, l'enseignant universitaire peut jouer un rôle important pour favoriser la motivation des étudiants. Tinto (2012) affirme que c'est la relation que vit l'étudiant avec son enseignant durant ses cours qui influence le plus son intégration académique et sociale, de même que sa motivation. Plusieurs auteurs démontrent également que les pratiques d'enseignement et d'évaluation ont un effet sur la motivation et sur la décision des étudiants à persévérer dans leurs études (Good et Brophy, 2000; Viau, 2009). De même, Crespo et Houle (1995) confirment que l'utilisation d'une stratégie pédagogique inadéquate se retrouverait parmi les principaux motifs d'abandon à l'université.

Les recherches portant sur les stratégies pédagogiques des enseignants universitaires sont pourtant peu nombreuses (Duguet, 2014) pour bien comprendre le phénomène. Les études recensées traitent davantage des effets de l'usage d'une stratégie pédagogique ou de la comparaison entre deux stratégies dans le cadre d'un même cours. Peu d'études ont documenté l'usage de stratégies multiples dans le cadre d'un même cours universitaire, bien qu'il apparaisse important d'en comprendre sa participation à la motivation des étudiants.

5 Plusieurs auteurs rapportent que l'enseignement magistral est la pratique la plus répandue en enseignement au postsecondaire (Darling-Hammond, 2006 ; Prégent et al., 2009). Or, les résultats d'une méta-analyse comparant la performance des étudiants dans les cours qui préconisent l'apprentissage actif et les cours traditionnels démontrent que les étudiants des classes utilisant l'enseignement magistral étaient 1,5 fois plus susceptibles d'échouer que les étudiants des classes utilisant l'apprentissage actif (Freeman et al., 2014).

D'autres enseignants utilisent des stratégies pédagogiques uniques, mais plus actives que l'enseignement magistral comme l'apprentissage par problèmes, l'approche par projets, etc., dont certaines recherches reconnaissent les bienfaits en pédagogie de 
l'enseignement supérieur (Huang et Wang, 2012; Viau, Joly et Bédard, 2004). L'utilisation systématique d'une même stratégie pédagogique dans tout le programme d'étude peut toutefois créer un effet de saturation chez les étudiants (Joyce, Weil et Calhoun, 2004, dans Legendre, 2005).

7 Ainsi, plusieurs chercheurs en éducation soutiennent l'importance d'utiliser différentes stratégies pédagogiques en enseignement (Halawah, 2011; Joyce et al., 2015 ; Pratt, 2005). Selon Sauvé et al. (2007), les activités pédagogiques doivent être diversifiées pour donner aux étudiants la possibilité de faire des choix et d'enrayer la routine. La recherche quantitative d'Halawah (2011), menée auprès de 232 étudiants universitaires ayant répondu à un questionnaire portant sur les facteurs influençant leur motivation, révèle l'importance d'utiliser une variété de stratégies pédagogiques pour capter l'attention des étudiants et susciter leur curiosité. Les résultats de cette recherche démontrent qu'en plus de la personnalité de l'enseignant, l'utilisation d'une variété de stratégies pédagogiques influence positivement la motivation à apprendre des étudiants. Ainsi, bien que le nombre minimal de stratégies pédagogiques à mobiliser pour parler de "diversification" demeure imprécis dans les écrits scientifiques, il semblerait qu'il soit préférable de recourir à une pluralité de stratégies pédagogiques pour profiter des avantages qu'offre individuellement chacune d'entre elles. Toutefois, à notre connaissance, aucune étude empirique ne s'est attardée à comprendre en profondeur les liens qui unissent la motivation des étudiants et l'utilisation de stratégies pédagogiques diversifiées à l'enseignement supérieur. Ainsi, notre question de recherche est la suivante : en quoi la diversité des stratégies pédagogiques participet-elle à la motivation à apprendre des étudiants ?

\section{Stratégies pédagogiques}

8 Plusieurs auteurs, dont Messier (2014), constatent un flou conceptuel et théorique en pédagogie. C'est le cas pour le concept de stratégie pédagogique, dont les appellations sont multiples. Dans sa thèse, Messier (2014) propose un réseau conceptuel qui précise et illustre les définitions des termes apparentés au terme "méthode » en pédagogie. Pour elle, une "stratégie pédagogique " se caractérise par une série d'opérations qui vise l'atteinte d'objectifs pédagogiques dans le cadre d'une situation pédagogique. L'enseignant doit choisir ou concevoir une stratégie pédagogique et la mettre en œuvre dans le cadre d'une situation pédagogique réelle.

9 Outre le concept de stratégie pédagogique, il convient de définir deux concepts spécifiques qui en découlent : "méthode pédagogique » et " technique pédagogique ». Pour qu'une stratégie pédagogique soit définie comme une méthode pédagogique, elle doit avoir été validée empiriquement ou scientifiquement et être connue et partagée " sous une appellation distincte » par les acteurs de la profession enseignante (Messier, 2014, p. 215). À titre d'exemple, l'enseignement explicite, l'apprentissage par problèmes et l'apprentissage par projets pourraient être qualifiés de méthodes pédagogiques parce qu'elles appartiennent à la culture propre de la profession enseignante et parce qu'elles se composent de phases qui les caractérisent. Quant à elle, la technique pédagogique est plutôt un "moyen" pédagogique utilisé par l'enseignant pour "atteindre un ou des objectifs pédagogiques spécifiques" (Messier, 2014, p. 222), moyen qui peut faire partie intégrante d'une méthode pédagogique ou qui peut être autonome. Par exemple, le graffiti collectif, utilisé en apprentissage coopératif 
(méthode pédagogique), est considéré comme une technique pédagogique. Également, le débat et le groupe de discussion, qui ne font pas nécessairement partie d'une démarche plus large sont aussi considérés comme des techniques pédagogiques dans le cadre de cette étude.

Plusieurs auteurs en éducation ont élaboré des typologies qui visent à classer les stratégies pédagogiques selon différents regroupements. Certains auteurs, dont Joyce et al. (2015), Raby et Viola (2016), de même que Vienneau (2017), privilégient un classement selon les courants de pensée en éducation. D'autres optent pour un classement selon le partage de l'initiative dévolue à l'enseignant ou à l'apprenant (Tournier, 1978) ou selon la centration sur l'apprentissage ou l'enseignement (magistocentrées, pédocentrées) (Chamberland, Lavoie et Marquis, 1995). Enfin, Prégent (1990) classe les stratégies pédagogiques en trois catégories (exposés, discussion/travail d'équipe, apprentissage individuel). Bref, ces différents classements contribuent à une meilleure compréhension de la nature de chacune des stratégies pédagogiques utilisées en enseignement au supérieur.

\section{Motivation à apprendre}

11 Différentes perspectives ont contribué à l'évolution du concept de motivation. Le courant sociocognitif de la motivation accorde une importance particulière au contexte et à l'environnement dans lequel l'étudiant apprend. Les théoriciens de l'approche sociocognitive considèrent que la motivation n'est pas innée et se construit au fil des expériences d'apprentissage et des interactions avec l'environnement. Plusieurs auteurs ont élaboré des modèles de motivation dans une perspective sociocognitive (Bandura, 1986 ; Eccles et Wiegfield, 2002 ; Pintrich et Schrauben, 1992 ; Viau, 2014). Ces modèles de la motivation ont alimenté les connaissances du domaine et ont contribué à l'évolution des théories de la motivation dans une perspective sociocognitive. Dans le cadre de cette recherche, le modèle de Viau (2014) apparaît le plus pertinent puisqu'il décrit la dynamique motivationnelle des étudiants en prenant comme point de départ les activités pédagogiques proposées par le professeur, dimension au cœur de la question de recherche abordée dans cet article. À cet égard, Viau considère que les activités pédagogiques favorisent l'engagement cognitif et la persévérance des étudiants. Dans son ouvrage qui porte entièrement sur la pédagogie de l'enseignement supérieur, il explique la dynamique motivationnelle au regard d'une activité pédagogique (Figure 1).

Figure 1. La dynamique motivationnelle au regard d'une activité selon Viau (2014, p. 238).

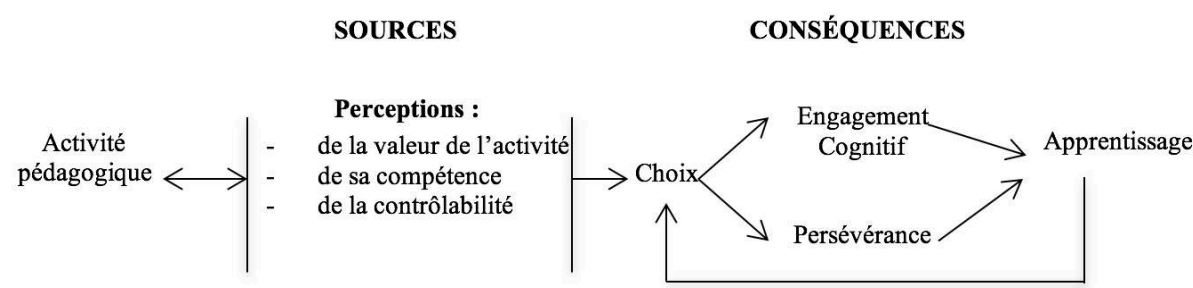

Ce modèle présente trois types de perceptions qui peuvent exercer une influence sur la dynamique motivationnelle de l'étudiant : sa perception de la valeur de l'activité, de sa 
compétence et de la contrôlabilité de l'activité. Plus spécifiquement, la perception de la valeur de l'activité réfère au jugement que l'élève porte sur l'activité qui lui est présentée. En fait, s'il est souhaité que l'apprenant s'engage cognitivement dans la réalisation de la tâche qui lui est demandée, la représentation cognitive de ce qu'il doit accomplir doit être claire pour l'apprenant. Quant à la perception de sa compétence, il s'agit pour l'étudiant d'évaluer ses capacités à accomplir l'activité de manière adéquate avant de la commencer. Si l'étudiant juge que ses capacités à réussir la tâche sont élevées, il sera plus engagé cognitivement et persévèrera dans la tâche. Enfin, la perception de contrôlabilité concerne le degré de contrôle qu'il aura au regard d'une activité à accomplir. Il est question ici du contrôle sur le déroulement, mais aussi sur les conséquences de l'activité proposée. Le choix, l'engagement cognitif, ainsi que la persévérance, sont les principales conséquences de la motivation à apprendre. Dans son modèle, la conséquence finale de la dynamique motivationnelle est l'apprentissage. La recherche de Viau et al. (2004) fait notamment le lien entre des stratégies pédagogiques qui stimulent intellectuellement les étudiants et la motivation à apprendre.

Qu'en est-il donc de l'utilisation diversifiée de stratégies pédagogiques dans le cadre d'un même cours universitaire sur la motivation à apprendre des étudiants? Nos objectifs de la recherche sont 1) de décrire l'utilisation et le contexte d'utilisation de stratégies pédagogiques diversifiées dans le cadre d'un cours universitaire et 2) d'examiner l'évolution de la motivation à apprendre des étudiants soumis à ses stratégies pédagogiques diversifiées à l'intérieur de ce même cours. L'étude s'appuie donc sur l'hypothèse que l'utilisation de stratégies pédagogiques diversifiées dans le cadre d'un cours universitaire participe à la motivation à apprendre des étudiants.

\section{Méthodologie}

14 Une étude a été menée auprès de quatre enseignants universitaires de deux domaines différents (génie et éducation) au premier cycle universitaire de trois universités au Québec. Un des enseignants de cette recherche s'est démarqué d'abord parce qu'il utilisait beaucoup plus de stratégiques pédagogiques dans le cadre de son cours que les trois autres, mais aussi par le fait que ses étudiants étaient les plus motivés à apprendre. C'est sur ce cas particulier que porte cet article. Les trois principales étapes de la méthode de l'étude de cas, proposée par Merriam (1988), ont été respectées dans cette étude : préparation, actualisation et interprétation.

\subsection{Participants et description sommaire du cas}

15 Un enseignant universitaire d'une université québécoise ainsi que les étudiants de première année d'un programme d'éducation préscolaire et d'enseignement primaire étant inscrit à son cours $(n=59)$ constituaient le cas à l'étude. L'échantillon contenait une très grande majorité de femmes étudiantes $(n=58)$, ce qui n'a rien d'exceptionnel dans ce type de programme.

$\mathrm{Au}$ moment de la collecte des données et à ce jour, Carlos ${ }^{2}$ occupe un poste de professeur universitaire en didactique des sciences humaines. Il est dans la fin trentaine et enseigne depuis près de 20 ans (enseignant au primaire et au secondaire, conseiller pédagogique, chargé de cours vacataire, professeur, etc.). Sur le plan de sa formation académique, il détient un baccalauréat en pédagogie. Il a une maitrise en 
sciences de l'éducation et un doctorat en éducation. Enfin, il est important de spécifier que Carlos a enseigné le cours dont il est question dans la présente étude à cinq reprises.

Pour sélectionner ce professeur, plusieurs étapes ont été nécessaires. D'abord, Carlos a été recommandé par sa directrice de programme parce que ses étudiants ont produit des évaluations positives de son enseignement et parce qu'il utilisait des stratégies pédagogiques diversifiées dans son enseignement. A la suite d'un entretien téléphonique, il est apparu que ce professeur utilisait au moins sept stratégies différentes dans son cours, ce qui était supérieur aux autres enseignants interrogés. Bien qu'il n'y ait pas de seuil minimal connu quant au nombre de stratégies utilisées pour pouvoir qualifier un enseignement de diversifié, l'enseignement magistral domine largement à l'université si bien que sept semblait déjà beaucoup. Dans une perspective de compréhension, de description et de découverte, au sens où l'entend Merriam (1988), ce professeur correspondait au profil recherché.

\subsection{Mesures}

18 Plusieurs outils de collecte de données (questionnaires, observations, entretiens semidirigés) ont été utilisés pour étudier en quoi les stratégies pédagogiques diversifiées utilisées dans le cours participent à la motivation à apprendre des étudiants.

D'abord, un questionnaire élaboré par Bédard et Viau (2001) comportant neuf items (ayant un alpha de Cronbach supérieur à .80) sur une échelle de réponse de type Likert ( 1 : Totalement en désaccord ; 2 : Plutôt en désaccord ; $3:$ Plus ou moins en accord; 4 : Plutôt d'accord et 5 : Totalement en accord) a été administré à trois reprises (cours $3,8$ et 13$)$ à tous les étudiants du groupe $(n=59)$. Ce questionnaire portait sur les perceptions de la valeur des activités proposées (p. ex. : les activités me sont utiles pour apprendre efficacement la matière), de compétence (p. ex.: je me sens capable de comprendre la matière) et de la contrôlabilité des activités proposées (p. ex.: j’ai le sentiment d'avoir un certain contrôle sur le déroulement des activités). Le questionnaire prenait environ cinq minutes à répondre en début de cours.

Trois observations en salle de classe à l'aide d'une grille d'observation ont aussi été réalisées (cours 8,10 et 13). Les séances d'observation ont été planifiées à l'avance avec le professeur. Cette modalité de fonctionnement est en accord avec la posture interprétative/compréhensive de la recherche puisqu'elle a permis de cibler les cours les plus susceptibles de proposer des stratégies pédagogiques diversifiées. La grille d'observation permettait de relever des informations sur l'environnement physique de la classe (type de local, positionnement du professeur dans le local, etc.), sur les habiletés à communiquer du professeur (communication verbale, communication corporelle, etc.) et sur le climat général de la classe (participation des étudiants, départs hâtifs, etc.). La grille permettait aussi de noter des commentaires en lien avec l'utilisation des stratégies pédagogiques (p. ex.: le professeur explique le jeu, les étudiants écoutent, etc.) et en lien avec le contexte (p. ex.: un grand groupe, un environnement physique exigu, etc.).

21 Un entretien semi-dirigé d'environ une heure avec le professeur s'est tenu à la fin de la session d'hiver 2016. Les questions visaient notamment à obtenir des précisions quant au contexte d'enseignement et d'apprentissage et à ce qui avait été observé afin de mieux décrire les stratégies pédagogiques ainsi que leur lien avec la motivation à 
apprendre des étudiants. Plus spécifiquement, la première partie du canevas d'entretien portait sur des éléments visant à décrire le cas (p. ex. : Depuis combien d'années enseignez-vous? Dans quelle discipline enseignez-vous? etc.). La deuxième partie du canevas portait sur l'objet d'étude et comportait des questions relatives à l'utilisation des stratégies pédagogiques, à leur contexte d'utilisation et à la motivation à apprendre des étudiants (p. ex.: Pourriez-vous décrire en détail les stratégies pédagogiques que vous avez utilisées durant la session dans le cadre de ce cours en particulier en décrivant chacune des activités réalisées?; Qu'est-ce qui peut influencer la motivation à apprendre de vos étudiants dans ce cours ; etc.).

Dix entretiens semi-dirigés d'une durée variant entre 14 et 25 minutes ont été menés avec des étudiants aux profils motivationnels différents : forte $(n=6)$ et faible $(n=1)$ motivation à apprendre ; augmentation $(\mathrm{n}=2)$ et diminution $(\mathrm{n}=1)$ de la motivation à apprendre au cours de la session. La sélection de ces étudiants s'est basée sur l'écart entre la moyenne de la motivation de la première et de la troisième passation du questionnaire. Des questions relatives à leur motivation à apprendre et au contexte ont d'abord été adressées aux étudiants (p. ex. : Décrivez-moi votre motivation à apprendre dans ce cours en début de la session, au milieu de la session, à la fin de la session; Décrivez-moi le climat de la classe dans ce cours. Etc.). D'autres questions portaient sur l'influence de l'utilisation des stratégies pédagogiques sur leur motivation à apprendre et sur les autres facteurs qui pouvaient l'influencer (p. ex.:Quelles stratégies pédagogiques utilisées par Carlos vous ont le plus motivé? Le moins motivé? Démotivé ?; D'autres facteurs ont-ils contribué à vous motiver ou à vous démotiver durant ce cours? ; etc.).

\section{Résultats}

Rappelons que le cas de Carlos et de ses 59 étudiants fait l'objet de cet article parce qu'à l'issue de l'analyse des données, il se distingue des autres cas au plan des stratégies pédagogiques utilisées et du niveau de motivation à apprendre des étudiants.

\subsection{Utilisation de stratégies pédagogiques diversifiées}

Afin de décrire les stratégies pédagogiques utilisées par Carlos, des analyses qualitatives issues des entretiens et des observations ont été menées. Pour ce faire, une grille de codage mixte (Van Der Maren, 2003) avec l'utilisation de codes émergents a été privilégiée. Cette grille d'analyse a d'abord été construite en fonction du cadre conceptuel. À la suite du codage de l'ensemble des données récoltées, un codage inverse a été réalisé afin de vérifier si chaque segment appartenait bien à la catégorie dans laquelle il avait été placé. Une liste de 162 codes a été établie et classée en trois grands thèmes, dont celui qui nous intéresse ici: les stratégies pédagogiques utilisées par Carlos. Au terme de ce travail d'analyse, il a été établi que Carlos utilisait 14 stratégies pédagogiques différentes dans son enseignement (voir le tableau 1).

Tableau 1. Les stratégies pédagogiques utilisées par Carlos.

Stratégies pédagogiques
Nombre d'extraits codifiés 


\begin{tabular}{|c|c|}
\hline Ateliers & 27 \\
\hline Jeu pédagogique & 19 \\
\hline Exposé magistral & 11 \\
\hline Présentation orale des étudiants & 11 \\
\hline Exposé interactif & 9 \\
\hline Retour & 8 \\
\hline Présentation d'une vidéo & 5 \\
\hline Travail d'équipe & 4 \\
\hline Jeu de rôles & 3 \\
\hline Apprentissage par problèmes & 2 \\
\hline Débat & 2 \\
\hline Étude de cas & 1 \\
\hline Groupe de discussion & 1 \\
\hline Travail individuel en classe & 1 \\
\hline
\end{tabular}

Les stratégies pédagogiques utilisées par Carlos sont diverses, tant au niveau du degré de contrôle de l'apprentissage et des courants pédagogiques qu'au niveau des techniques et des méthodes utilisées. Une majorité de stratégies pédagogiques sont pédocentrées (p. ex. : apprentissage par problèmes), alors que d'autres sont plutôt magistrocentrées (exposé magistral, exposé interactif, présentation d'une vidéo, etc.). Plusieurs stratégies sont inspirées du courant socioconstructiviste (p. ex. : jeu de rôles), mais également béhavioriste (p. ex. : exposé magistral). Peu de stratégies pédagogiques sont inspirées du courant cognitiviste (travail individuel en classe) et aucune du courant humaniste de l'apprentissage. Les stratégies pédagogiques utilisées par Carlos comprennent à la fois des méthodes (p.ex.: étude de cas) et des techniques pédagogiques (p. ex. : jeu de rôles).

Ces stratégies pédagogiques identifiées en nombre d'extraits codés de façon qualitative dans le tableau 1 ont également été comparées à l'aide d'un test Chi-carré, qui permet de vérifier si chacune des stratégies est significativement plus utilisée que les autres. Les résultats $d u$ test révèlent que certaines stratégies sont significativement plus populaires que d'autres $\left(\chi^{2}(13)=103,133, p<0,001\right)$. Ce résultat vient confirmer la grande diversité des stratégies de Carlos, mais apporte également une compréhension des particularités pédagogiques du professeur. Les résultats qualitatifs permettent d'identifier que les stratégies les plus rapportées sont l'atelier, le jeu pédagogique et la présentation orale par des étudiants. A l'inverse, le travail individuel, l'étude de cas, le groupe de discussion, le débat et l'apprentissage par problèmes sont celles dont les participants parlent le moins. Un autre résultat intéressant concerne l'enseignement 
magistral, dont le nombre de fois que cette stratégie a été mentionnée est relativement moyen par rapport aux autres stratégies pédagogiques. En outre, une autre forme d'exposé magistral que privilégie Carlos est la présentation orale donnée par les étudiants. Bien qu'il s'agisse d'une forme d'enseignement magistral, cette stratégie place toutefois les étudiants au cœur de cette pratique. A la suite des résultats de ce test statistique et de l'analyse des données qualitatives, certaines stratégies ressortent du lot parce que les étudiants en font mention lorsqu'ils expliquent ce qui les motive particulièrement dans le cours.

Une des stratégies que Carlos utilise est le jeu pédagogique. Cette stratégie fait participer les étudiants: "J'ai aimé ses petits jeux-questionnaires, l'animation qu'il faisait c'était bien, parce que ça faisait participer le groupe qui se réveillait un peu le vendredi matin ». Les observations en salle de classe appuient aussi ce constat en plus de mettre en lumière le plaisir que leur procure cette stratégie. A la question concernant les activités qui avaient été motivantes, une étudiante a répondu : «Dans le fond, les jeux. Je pense que c'est toujours un bon moyen de capter l'attention des gens ». Carlos fait des retours sur les lectures obligatoires en mettant à profit sa passion pour les jeux de société et les jeux télévisés. Les retours sur les lectures sont donc abordés de façon " plaisante » et " participative », ce qui semble plaire à la majorité des étudiants reçus en entretien (7/10). Carlos commence par une introduction, exactement comme dans le jeu télévisé. Ensuite, il sollicite la participation des étudiants en invitant par exemple deux étudiants à venir à l'avant. L'un ou l'autre des adversaires doit répondre rapidement à la question posée sur les lectures après avoir appuyé sur la clochette. Chaque question est ensuite retournée au groupe pour qu'ils trouvent les autres réponses possibles et/ou complémentaires. Lors de cette activité, les étudiants s'encouragent, s'applaudissent ». Enfin, Carlos prend le temps de faire des parenthèses théoriques après chaque question et les étudiants prennent des notes et posent des questions.

Carlos fait beaucoup travailler ses étudiants en équipe, et cela de différentes façons. En classe, il prévoit des ateliers où il fait créer des situations d'apprentissage. Une étudiante mentionne en entretien que "c'était plaisant. Ça nous donnait plein d'idées et c'était vraiment dans le concret. [...]. Ça nous mettait en pratique». Les ateliers permettent aux étudiants d'appliquer les notions vues en début de cours et de bâtir des situations d'apprentissage pour leur future classe. Lors de ces périodes de travail en équipe, Carlos circule fréquemment pour aider les étudiants, il répond aux questions et leur donne des explications plus précises au besoin.

Également, le professeur utilise le débat lors d'un de ses cours. Il soumet des phrases à deux groupes en lien avec l'éducation à la citoyenneté. Les équipes doivent se concerter et donner des arguments pour appuyer leur position préalablement définie par le professeur (pour ou contre).

30 Pour la plupart des activités réalisées en équipe pendant le cours, Carlos forme luimême les équipes et varie les façons de faire. Également, le professeur fournit le matériel nécessaire à la réalisation de l'activité, ce qui semble très apprécié des étudiants. Il peut s'agir de crayons, d'affiches, de textes et même de matériel didactique en univers social. Selon les étudiants reçus en entretien, le fait de fournir le matériel contribue à diminuer la pression et le stress chez les étudiants.

31 Une grande partie du cours est consacrée aux présentations orales des étudiants en équipe. Trois étudiantes qui ont été présentes à tous les cours où il $\mathrm{y}$ a eu des exposés 
mentionnent avoir trouvé cette stratégie utile: «De voir les autres équipes qui présentaient, ça me donnait vraiment un large éventail d'activités ». Carlos prend des notes et est à l'écoute lors des présentations. À la fin des exposés, il pose des questions aux équipes et adresse une question synthèse à l'ensemble des étudiants. Il formule aussi des pistes d'interprétation.

Carlos propose aussi plusieurs activités et exercices à réaliser seul ou en dyades pendant le cours. Parfois, une activité de type jeu de rôle est proposée aux étudiants. Par exemple, les étudiants doivent se mettre à la place d'un élève du primaire pour analyser la diversité entre la société algonquienne et iroquoise vers 1905. Après avoir réalisé l'activité en tant qu'apprenants, les étudiants doivent identifier les difficultés ressenties par l'élève. Ensuite, ils doivent se placer à la place de l'enseignant et cibler la meilleure façon de rendre une telle analyse accessible aux élèves. Après l'activité de jeu de rôle, le professeur anime une plénière, ce qui offre l'occasion aux étudiants de partager leur travail à l'ensemble du groupe.

Également, le professeur place ses étudiants dans une situation qui pourrait réellement se produire en classe. Par exemple, en suivant quelques étapes de l'apprentissage par problèmes, les étudiants doivent expliquer ce qu'ils feraient s'ils étaient engagés dans une école comme enseignant de sciences humaines. Dans le même sens, lors d'une séance de cours portant sur la planification et l'évaluation des apprentissages, Carlos propose aux étudiants une mise en situation les invitant à préparer une planification globale pour l'année scolaire (compétences, savoirs essentiels et progression des apprentissages), comme ils auront à le faire dans le cadre de leurs tâches d'enseignants au primaire.

34 Enfin, Carlos utilise un des cas qu'il a observé dans le cadre de ses activités de recherche pour illustrer un phénomène à l'étude dans le cours. Par exemple, les cas de trois enseignantes utilisant respectivement un style transmissif d'enseignement, une pédagogie de la découverte et une démarche à caractère scientifique/constructiviste sont analysés et comparés entre eux par les étudiants pendant le cours. Les cas présentés par écrit présentent des enseignantes qui donnent une leçon portant sur « les écoles d'ici et d'ailleurs» pour travailler les concepts d'espace, de société et de microsociété-école. Cette activité permet aux étudiants d'envisager trois manières différentes d'enseigner un même concept.

Pour faire des synthèses et des retours sur les activités, aux dires des étudiants, Carlos utilise l'enseignement magistral et l'exposé interactif. Ces stratégies sont utilisées sur une courte période durant le cours. Le professeur n'y voit que des bienfaits lorsqu'il est question de faire des synthèses: "C'est l'exposé que j'utilise rarement. Celui-là, je trouve que les étudiants vont regarder plus sur leur téléphone. Ils sont un peu plus distraits, mais pour moi ça prend quand même certains blocs où c'est moi qui fais une synthèse. C'est moi qui prends la parole pour mettre certains éléments au clair. Définir certains concepts ou revenir sur certains éléments. [...] Pour moi, c'est essentiel cette stratégie-là. »

C'est souvent à la suite d'une activité vécue en classe ou d'une lecture réalisée à la maison que Carlos fait un retour. Il explique à cette occasion l'utilité de l'activité et en quoi elle pourrait être réinvestie dans la future profession des étudiants. Aussi, Carlos utilise parfois l'exposé interactif durant ses cours et prévoit des moments d'échanges et de questionnements avec les étudiants. Il utilise très peu les présentations de type PowerPoint lors de ses exposés. Il fait plutôt des lignes du temps pour situer des 
évènements sur le plan chronologique à l'aide d'affiches. Il utilise aussi les schémas pour situer les liens entre différents concepts étudiés ou une carte conceptuelle trouée où les étudiants doivent trouver les mots manquants.

\subsection{Motivation à apprendre des étudiants}

Des analyses descriptives (moyennes et écarts-types) ainsi que des tests statistiques (ANOVA à mesures répétées) ont été réalisés d'emblée afin d'examiner l'évolution de la motivation à apprendre des étudiants. Une analyse factorielle exploratoire a permis de s'assurer que trois composantes distinctes (perception de la valeur de l'activité, perception de compétence et perception de la contrôlabilité) étaient mesurées à l'aide du questionnaire. Pour chacun des temps de mesure, seuls les étudiants ayant complété au moins $80 \%$ des items du questionnaire ont été retenus pour les analyses. Le tableau 2 présente l'évolution de la motivation des étudiants dans le temps.

Tableau 2. Moyenne (Écart-type) des trois sources de la motivation et de la motivation à chacun des temps

\begin{tabular}{|l|l|l|l|}
\hline $\begin{array}{l}\text { Trois sources de la dynamique motivationnelle et } \\
\text { motivation }\end{array}$ & T1 & T2 & T3 \\
\hline Perception de la valeur de la tâche & $\begin{array}{l}4,17 \\
(0,63)\end{array}$ & $\begin{array}{l}4,13 \\
(0,80)\end{array}$ & $\begin{array}{l}4,29 \\
(0,72)\end{array}$ \\
\hline Perception de compétence & $\begin{array}{l}4,37 \\
(0,52)\end{array}$ & $\begin{array}{l}4,36 \\
(0,49)\end{array}$ & $\begin{array}{l}4,44 \\
(0,57)\end{array}$ \\
\hline Perception de contrôlabilité & $\begin{array}{l}3,83 \\
(0,69)\end{array}$ & $\begin{array}{l}3,88 \\
(0,68)\end{array}$ & $\begin{array}{l}4,03 \\
(0,78)\end{array}$ \\
\hline Moyenne de la motivation dans le temps & $\begin{array}{l}4,16 \\
(0,48)\end{array}$ & $\begin{array}{l}4,15 \\
(0,57)\end{array}$ & $\begin{array}{l}4,28 \\
(0,60)\end{array}$ \\
\hline
\end{tabular}

Tel que représenté dans le tableau 2, les étudiants du groupe de Carlos étaient particulièrement motivés à apprendre $(\mathrm{T} 1: 4,16 ; \mathrm{T} 2: 4,15 ; \mathrm{T} 3: 4,28$, chaque indice sur une échelle de 5).

Pour réaliser les analyses quantitatives, les items relatifs à chaque composante ont d'abord été combinés pour en faire une moyenne à chacun des trois temps de mesure. Ces moyennes ont ensuite été comparées entre elles avec une ANOVA à mesures répétées pour examiner si les trois sources motivationnelles retenues évoluaient différemment dans le temps. Les résultats de l'analyse multivariée ont révélé que la motivation ne fluctuait pas significativement dans le temps $F(6,33)=0,63, p=0,704, \eta^{2}$ $=0,10$. Ces résultats s'expliquent notamment par le fait que la motivation des étudiants était déjà élevée au premier temps de mesure et qu'elle est demeurée relativement stable dans le temps. D'ailleurs, un examen détaillé des résultats individuels obtenus a révélé que sur les 39 étudiants ayant répondu aux trois temps de mesure, deux ont maintenu un degré de motivation identique entre le $1^{\text {er }}$ temps et le $3^{\mathrm{e}}$ temps de mesure alors que la motivation a augmenté pour 24 étudiants. En d'autres termes, les données 
révèlent un déclin de la motivation pour 13 étudiants entre le $1^{\text {er }}$ et le $3^{\mathrm{e}}$ temps de mesure.

En plus de ces résultats quantitatifs, les analyses qualitatives issues des entretiens et des observations en salle de classe révèlent des résultats éclairants. Au terme des analyses, il s'avère que 21 extraits témoignent du haut niveau de motivation, huit d'une motivation variable et deux extraits du peu de motivation des étudiants. Les étudiants motivés mentionnent qu'ils étaient contents d'être au cours, que «la motivation était pas mal générale » au sein du groupe. Une étudiante mentionne « c'est un cours que j'ai vraiment aimé. Je l'ai trouvé vraiment motivant ", et ce, «même si c'était des cours de quatre heures ». L'implication attendue des étudiants dans le cours "forçait à être motivé ». Une étudiante soutient même que « c'est l'un des meilleurs cours que j'ai eu à ce jour !». Pour ceux qui trouvent que leur motivation a varié entre le début et la fin de la session, ils expliquent que c'est surtout en fin de session que leur motivation a baissé, principalement parce que les derniers cours alimentaient moins le contenu à intégrer aux travaux de session. D'autres étudiants ont commencé la session moins motivés parce qu'ils avaient des "appréhensions", mais une étudiante mentionne qu'elle est » contente d'avoir eu Carlos comme enseignant». Elle complète en mentionnant que Carlos offre des cours avec des travaux pratiques. Pour les deux étudiantes qui mentionnent avoir été peu motivées durant le cours, elles spécifient que certains cours étaient moins " pertinents » ou " intéressants », qu'ils « n'apportaient rien de plus pour les apprentissages ».

41 Plus précisément, des résultats au niveau des trois sources de la motivation ont également émergés de l'analyse qualitative des données. Ces trois sources de la motivation semblent avoir participé à certains égards à la motivation à apprendre des étudiants.

D'abord, 52 extraits ont été codés en lien avec la perception de la valeur de la tâche. Les étudiants ont mentionné que le fait de faire des liens avec la pratique en milieu scolaire est très important. Par exemple, Carlos propose des vidéos d'enseignantes en exercice qui appliquent les notions enseignées ou il donne des exemples concrets en analysant du matériel pédagogique. Les étudiants trouvent qu'il offre un cours « très appliqué » et qui « n'était pas complètement déconnecté ou juste du par cœur ». Le fait de savoir que l'activité ou la notion à apprendre peut facilement être réinvestie dans la pratique semble motiver les étudiants. Pour eux, il est important que ce qui est présenté en classe soit pertinent pour le stage à venir et pour leur future profession. Enfin, Carlos fait des synthèses, des retours et des plénières à la fin des activités qu'il propose, ce qui semble aider les étudiants à comprendre comment réinvestir les contenus appris dans la pratique.

Quant à la perception de contrôlabilité, la plupart des commentaires se rapportent à l'évaluation de fin de session (huit extraits). Les étudiants apprécient le fait de pouvoir choisir les modalités de réalisation de l'examen : "c'était vraiment libre ». Carlos offre également d'autres choix aux étudiants dans ses cours, dont la possibilité de travailler à l'extérieur du local d'enseignement lors des ateliers et celle de choisir le format que prennent les présentations orales.

44 Enfin, il semble que la perception de compétence soit moins importante pour les étudiants puisqu'ils n'en font mention qu'à deux occasions lors des entretiens individuels. Ce cours semble les avoir mis en confiance avec les tâches d'enseignement qu'ils auront à accomplir. À cet égard, une étudiante mentionne qu'elle se «sentirait 
quand même apte à enseigner un cours d'univers social demain ». Or, les séances d'observation en salle de classe ainsi que l'entretien avec le professeur tendent à révéler que le professeur pose des gestes pour favoriser le sentiment de compétence des étudiants ; sept extraits ont été codés en ce sens. Carlos prend le temps de féliciter les étudiants lorsqu'ils donnent de bonnes réponses. De plus, il les rassure sur les délais de réalisation des travaux.

\subsection{Enseignement diversifié et motivation à apprendre des étudiants}

45 L'éventail des stratégies pédagogiques utilisées par Carlos durant la session a vraisemblablement contribué à la motivation des étudiants du cours (39 extraits). En fait, le cours offert par Carlos constitue un cas particulier selon une étudiante: "Ça faisait un changement des cours typiques de l'université ». Le fait qu'il utilise des stratégies variées "changeait beaucoup la dynamique d'un cours universitaire». Lorsque « c'est trop pareil, c'est vraiment démotivant, surtout pour la longueur de cours qu'il devait donner. C'[était] pertinent qu'il le fasse ». Selon Carlos, l'utilisation de stratégies pédagogiques diversifiées permet de soutenir l'attention et de favoriser la participation des étudiants : «Ce sont des activités qui permettent quand même aux étudiants de participer. Il y a plusieurs étudiants qui autrement ne prendront pas la parole en classe ". À cet égard, les résultats qualitatifs mettent en évidence le fait que Carlos met ses étudiants en action (11 extraits) et les fait travailler en équipe (10 extraits). Une étudiante souligne que « Carlos avait un défi de taille parce qu'on était 60 dans un petit local cordé. Puis ce n'était pas toujours évident. On aurait tendance à croire que dans ce local-là, le magistral allait l'emporter ». Une étudiante soutient que la façon d'enseigner de Carlos est " très variée ", qu'« il utilisait plusieurs pédagogies. Il était toujours dans le socioconstructivisme. Il utilisait beaucoup de façons différentes d'apporter la nouvelle matière "; " Ça nous permettait vraiment de mieux apprendre, d'apprendre en faisant quelque chose ». Bref, Carlos utilise plusieurs stratégies pédagogiques pendant son cours en accordant une place importante aux stratégies qui mettent les étudiants en action.

Aucun étudiant n'a mentionné d'éléments négatifs reliés à l'utilisation de stratégies pédagogiques diversifiées. Par contre, certains obstacles reliés à une utilisation diversifiée des stratégies ont été soulevés par le professeur. Notamment, Carlos souligne les coûts importants que les stratégies qu'il utilise dans ce cours engendre pour lui (p. ex. : l'achat de matériel pédagogique), en plus de lui demander davantage d'organisation et un temps supplémentaire pour la planification de son enseignement (six extraits). Enfin, les conditions administratives ne favorisent pas l'apprentissage actif en contexte d'enseignement universitaire selon lui. À titre d'exemple, la réalisation d'une situation d'apprentissage par problèmes en entier est pratiquement impossible selon Carlos, si l'on souhaite respecter toutes les étapes sans avoir d'auxiliaires d'enseignement. 


\subsection{Qualités personnelles du professeur et motivation à apprendre des étudiants}

Malgré que les qualités personnelles du professeur ne fassent pas partie des objectifs de cette étude, leur contribution à la forte motivation à apprendre des étudiants mérite d'être soulignée (79 extraits). Entre autres, la personnalité et le style d'enseignement $\mathrm{du}$ professeur (17 extraits) sont mentionnés à plusieurs reprises par les étudiants comme ayant contribué à leur motivation durant le cours. Une étudiante a trouvé que c'était «vraiment un bon professeur » et une autre spécifie que sa personnalité «a contribué à faire en sorte, que [...] ça répondait à [son] style d'apprentissage ». De plus, la capacité du professeur à établir une relation de confiance (15 extraits) avec ses étudiants est importante. Carlos réussit à établir des liens avec ses étudiants, notamment en démontrant de l'intérêt pour leur parcours de formation : «il montrait beaucoup qu'il était intéressé par notre cheminement. Souvent, il s'informait de la session intensive : Comment ça se passe ? Est-ce que vous y arrivez?». En ce sens, il a su créer un bon contact avec eux en instaurant un climat de confiance et en limitant le plus possible la relation de hiérarchie qui existe entre professeur d'université et étudiant : « il a vraiment établi un respect mutuel, dès le départ, mais sans s'imposer en tant que figure d'autorité ». Également, dix extraits révèlent que Carlos a un grand sens de l'humour. À ce niveau, une étudiante mentionne qu' : « autant le professeur que les étudiants pouvaient faire des blagues». Un autre élément ressort en lien avec les qualités du professeur, selon les étudiants, Carlos fait preuve d'une grande disponibilité (neuf extraits), surtout lorsque les étudiants lui posent des questions : « il nous laissait savoir qu'on pouvait aller le voir pour lui poser des questions». Les étudiants mentionnent également que la compétence du professeur et son expérience du domaine contribuent à leur motivation (sept extraits). Enfin, dans une moindre mesure, il s'avère que le dynamisme du professeur (quatre extraits), le fait qu'il ne soit pas trop sévère (quatre extraits), qu'il donne beaucoup de rétroaction (trois extraits) et qu'il fasse preuve d'humilité (trois extraits) ont contribué aussi à la motivation à apprendre des étudiants.

\section{Discussion}

\subsection{Utiliser des stratégies pédagogiques diversifiées pour motiver ses étudiants}

Les données détaillées confirment que les nombreuses stratégies pédagogiques utilisées par Carlos (14) contribuent au niveau élevé de la motivation des étudiants de son cours. Ainsi, Carlos crée des situations pédagogiques qui suscitent l'engagement cognitif des étudiants, là où la plupart des enseignants gardent les étudiants beaucoup moins actifs dans leurs apprentissages. Ce choix a une grande influence sur le niveau de participation et de collaboration des étudiants en classe. Ces résultats corroborent ceux de l'étude d'Halawah (2011) sur les facteurs qui influencent la motivation à apprendre des étudiants universitaires, qui démontre que la variété des stratégies pédagogiques est importante pour les étudiants et que l'enseignement magistral ou l'exposé interactif ne doivent pas être les seules stratégies à privilégier. Il est important de trouver des stratégies alternatives qui seront en mesure de capter l'attention et la curiosité des 
étudiants pour d'éviter l'effet de saturation que peut entrainer l'utilisation répétitive d'une même stratégie pédagogique (Joyce et al., 2015). Nous avons constaté que les avantages liés à la diversification des stratégies pédagogiques sont étroitement liés à la participation soutenue des étudiants et à leur apprentissage. D'ailleurs, l'étude de Bédard et Viau (2001) révèle que les stratégies qui favorisent le plus l'apprentissage sont des stratégies participatives: l'approche par projets, l'étude de cas et l'approche par problèmes.

Les résultats mettent également en lumière la volonté de changement et de diversification des stratégies pédagogiques, tout en conservant une certaine part d'enseignement plus traditionnel ; ce qui va dans le sens du changement de paradigme qui semble s'opérer en enseignement supérieur ces dernières années (Ménard, 2017). En effet, les résultats de l'étude de Ménard (2017), menée auprès de 22 professeurs de trois universités canadiennes, révèlent que les pratiques observées et déclarées dépassent la transmission de contenu et sont maintenant plus centrées sur l'apprentissage des étudiants.

De plus, dans le contexte où le cours offert par Carlos s'adressait à des futurs enseignants à l'éducation préscolaire et en enseignement primaire, l'utilisation diversifiée de stratégies pédagogiques paraît encore plus importante que pour d'autres disciplines universitaires. À ce propos, les résultats énoncés révèlent que ce cours se distinguait des autres. L'éventail des stratégies utilisées par Carlos est jugé d'autant plus pertinent si l'on considère le peu de contact que ces étudiants auront avec les différentes stratégies pédagogiques dans leur formation. En effet, Carlos est un acteur important de la formation initiale des maîtres et joue un rôle de premier plan dans l'appropriation et l'utilisation de stratégies pédagogiques diversifiées par les futurs enseignants (Tremblay-Wragg, 2013).

51 Au terme des analyses qualitatives des données, aucun désavantage n’a été soulevé par les étudiants à propos de la diversification des stratégies pédagogiques et leur motivation. Or, les contraintes sont davantage reliées à des aspects organisationnels qui peuvent influencer la décision des professeurs d'utiliser des stratégies pédagogiques diversifiées (coûts importants pour le professeur selon les stratégies utilisées, planification plus longue, organisation plus lourde, etc.). À cet égard, plusieurs auteurs (Leclerc, Bourassa et Macé, 2017; Prégent et al., 2009) soulèvent le tiraillement entre l'enseignement, la recherche et les tâches administratives que vivent continuellement les professeurs à l'université. L'enseignement étant l'aspect le moins valorisé dans la carrière professorale actuellement, cela contribue probablement à un moindre investissement de leur part en la matière.

Ensuite, les résultats de cet article témoignent d'une situation d'enseignement où la motivation des étudiants est très élevée à chacun des temps de passation du questionnaire. L'étude de Bédard et Viau (2001) réalisée auprès de 4820 étudiants universitaires rapporte des scores moins élevés allant de 3,61 à 4,28 sur 5 selon l'utilisation d'une stratégie pédagogique (approche par projets : 4,28; étude de cas: 4,11 ; apprentissage par problèmes : 4,05; atelier : 4,01; séminaire de lecture : 3,61). Il faut toutefois analyser ces résultats avec prudence puisque l'étude de Bédard et Viau (2001) portait sur plusieurs cours d'un programme de formation dispensés par différents professeurs alors que la nôtre se déroulait dans un même cours avec le même professeur. Ce constat implique en lui-même une réflexion intéressante quant à la 
diversification des stratégies pédagogiques dans un même cours comparativement à une utilisation diversifiée dans un curriculum de formation.

Également, l'absence de diminution de la motivation à apprendre des étudiants de Carlos constitue en soi un résultat encourageant. Les résultats de l'étude de Ménard et Leduc (2016) s'intéressant à la motivation à apprendre des étudiants de français et de littérature au cégep témoignent d'une décroissance de la motivation pendant le trimestre dans la grande majorité des cas et du peu de groupes maintenant ou accroissant leur motivation à apprendre. Les résultats de cette recherche suggèrent que l'on observe habituellement une baisse de motivation au courant d'une même session, alors que le niveau de motivation s'est maintenu ou a augmenté pour seulement 7 groupes sur 48. Dans le cas présent, nous avons constaté que la motivation des étudiants de Carlos est restée assez stable au cours de la session, ou du moins, qu'elle n'a pas diminué. Les résultats individuels ont également démontré que la majorité des étudiants ayant répondu aux trois temps du questionnaire ont maintenu ou augmenté leur motivation à apprendre entre le début et la fin du cours dispensé par Carlos.

\subsection{Le lien entre les qualités personnelles du professeur et la motivation à apprendre des étudiants}

Comme la recherche d'Halawah (2011), nos résultats témoignent de l'importance des qualités personnelles du professeur. Selon cet auteur, l'ouverture, la capacité d'entrer en relation, l'enthousiasme, de même que la connaissance du nom de ses étudiants et de leurs intérêts sont les qualités personnelles d'un professeur qui favorise le plus la motivation des étudiants. S'intéresser aux étudiants contribue à développer une relation de confiance avec eux. Les résultats de notre recherche témoignent de la capacité de Carlos à établir une relation de confiance avec ses étudiants. L'étude réalisée par Bujold et Saint-Pierre (1996) révèle également que la qualité de la relation affective entre le professeur et les étudiants est importante pour produire des apprentissages plus durables chez les étudiants au premier cycle universitaire. Plusieurs indicateurs observables d'une relation affective positive énoncés dans l'article de Bujold et Saint-Pierre (1996) se rapprochent des résultats de notre étude, notamment en lien avec les caractéristiques personnelles de Carlos (est perçu comme étant intéressant et captivant par les étudiants, se soucie du succès de ses étudiants, est disponible, instaure une relation de confiance avec ses étudiants).

Finalement, il faut noter que le fait que les étudiants trouvent le cours plaisant, agréable et amusant a un effet positif sur leur motivation à apprendre. Les extraits présentés en lien avec les trois sources de la motivation font souvent référence au "plaisir» que ressentent les étudiants à suivre ce cours. Il est donc possible de se demander si cet aspect participe également à la motivation à apprendre des étudiants.

\subsection{Limites, pistes de recherches futures et retombées sur la pratique}

56 La présente étude comporte certaines limites qu'il convient d'énoncer et qui pourront guider la recherche future. D'abord, le fait que les étudiants avaient déjà un haut niveau de motivation est étonnant et pourrait être dû au fait que le niveau initial de motivation a été mesuré trop tard dans la session. Bien que cet aspect méthodologique 
puisse expliquer pourquoi les résultats n'ont pas permis de montrer une progression significative positive dans la motivation des étudiants de Carlos, il est également possible que les quelques cours suivis avec lui aient été suffisants pour accroitre la motivation des étudiants dès le début de la session, procurant ainsi un niveau élevé de motivation au premier temps de mesure.

Également, des éléments en lien avec les conditions physiques et organisationnelles d'enseignement auraient pu jouer sur le niveau de motivation des étudiants. Le local où enseignait Carlos lors des observations était situé dans le sous-sol d'un pavillon de l'université. Il n'était pourvu d'aucune fenêtre. Il était d'une capacité de 60 personnes alors que 59 étudiants étaient inscrits au cours. Le cours était offert en session intensive et d'une durée de quatre heures par séance. Nous aurions pensé que ces conditions d'enseignement auraient participé à une diminution de la motivation chez les étudiants du cours. Or, les données révèlent que le niveau de motivation est demeuré élevé malgré un contexte d'enseignement qui apparaissait loin d'être optimal.

En dépit de ces limites, les résultats de notre étude viennent combler un manque évident en ce qui a trait à la compréhension des liens qui unissent l'utilisation de stratégies pédagogiques diversifiées par des enseignants universitaires et la motivation à apprendre de leurs étudiants. Le cas de Carlos semble appuyer les propos des chercheurs en éducation (Halawah, 2011 ; Joyce et al., 2015 ; Pratt, 2005) qui soutiennent que la diversité des stratégies pédagogiques est importante. Sur cette base, des recherches ultérieures pour identifier les stratégies qui contribuent significativement à accroître la motivation des étudiants non seulement au premier cycle mais également aux études supérieures seraient hautement souhaitables.

De plus, il serait intéressant d'évaluer la motivation avant même l'amorce du cours pour avoir une mesure non teintée par le cours ou par l'enseignant. Des études ultérieures devraient reprendre le devis utilisé avec une mesure initiale précédant le début de la session, d'une part, et avec un groupe contrôle, d'autre part. À ce propos et afin d'évaluer la généralisation de ses résultats, il serait intéressant d'étudier ces mêmes questions dans d'autres contextes en analysant la motivation de plusieurs groupes qui reçoivent le même cours donné par le même professeur et ajouter au canevas d'entretien des questions spécifiques aux trois sources de la motivation énoncées par Viau (2014). Une autre suggestion serait de mesurer la motivation d'une cohorte d'étudiants ciblés dans tous leurs cours d'une même session et de comparer leur motivation entre ses différents cours en prenant en compte les stratégies pédagogiques utilisées par leurs enseignants.

60 Par ailleurs, les données produites dans cette étude peuvent avoir un impact non négligeable pour orienter les pratiques pédagogiques en vue de motiver davantage les étudiants universitaires et ultimement favoriser leurs apprentissages et leur réussite académique. Ces résultats pourraient amener les enseignants universitaires qui n'utilisent qu'une seule stratégie pédagogique dans leur cours à envisager la possibilité de varier leurs stratégies pédagogiques en complément aux pratiques plus traditionnelles. Ils sont maintenant mieux informés des gains potentiellement associés à l'utilisation de plusieurs stratégies pédagogiques dans le cadre d'un cours sur la motivation à apprendre des étudiants. 


\section{Conclusion}

Cette recherche portant sur la réussite et la persévérance scolaires des étudiants au premier cycle universitaire s'est intéressée à comprendre si l'utilisation diversifiée de stratégies pédagogiques participait à la motivation des étudiants. Plus spécifiquement, l'analyse en profondeur du cas d'un professeur d'université et de ses 59 étudiants a permis de décrire les stratégies pédagogiques utilisées par ce professeur et à examiner l'évolution de la motivation à apprendre de ses étudiants. Contrairement aux écrits disponibles qui se fondent typiquement sur l'étude d'une stratégie pédagogique ou sur la comparaison entre deux ou plusieurs stratégies, cet article adopte une approche nouvelle en faisant la description du cas unique d'un professeur d'université qui diversifie ses stratégies pédagogiques dans le cadre d'un même cours. Ce faisant, les résultats font ressortir des éléments qui sont susceptibles d'apporter une contribution importante à la motivation des étudiants à l'université. Les résultats des analyses quantitatives et qualitatives des données témoignent de l'utilisation diversifiée de stratégies pédagogiques dans son enseignement (14 stratégies au total) et de l'utilisation plus fréquente de certaines stratégies pédagogiques actives. Les résultats énoncés démontrent que la motivation à apprendre est restée élevée tout au long de la session et que plusieurs facteurs semblent y avoir contribué. A ce propos, les stratégies pédagogiques diversifiées utilisées ainsi que ses qualités personnelles agissent sur la motivation à apprendre de ses étudiants. La diversification des stratégies pédagogiques peut être freinée par certains obstacles, notamment les coûts associés à la façon dont Carlos a mis en œuvre certaines stratégies pédagogiques et le temps supplémentaire de planification nécessaire à leur conception. Or, les résultats démontrent qu'il est profitable de contrer ces obstacles, de placer au centre de ses préoccupations l'apprentissage des étudiants, et d'opter pour une diversité de stratégies pédagogiques pour maintenir une forte motivation à apprendre chez les étudiants tout au long du cours.

Ainsi, cette recherche offre aux acteurs et aux penseurs de l'éducation des résultats axés sur des données empiriques de recherche favorisant une réflexion plus poussée sur l'utilisation des stratégies pédagogiques diversifiées à l'université. C'est pourquoi, dans une visée plus large, cet article se veut un point de départ pour une réflexion plus en profondeur sur la pédagogie universitaire ayant comme finalité l'amélioration de la formation pédagogique des futurs enseignants en milieu universitaire et de ceux en exercice en vue de favoriser l'apprentissage et par conséquent la réussite et la persévérance scolaires des étudiants universitaires.

\section{BIBLIOGRAPHIE}

Altet, M. (2009). Professionnalisation et formation des enseignants. Dans J. Clanet (dir.), Recherche/formation des enseignants : quelles articulations (p. 19-32). Rennes: Presses universitaires de Rennes. 
Bandura, A. (1986). Social foundations of thought and action: A social cognitive theory. New Jersey: Prentice-Hall.

Bédard, D. et Viau, R. (2001). Le profil d'apprentissage des étudiantes et des étudiants de l'Université de Sherbrooke. Rapport de recherche. Sherbrooke : Université de Sherbrooke, Vice-rectorat à l'enseignement.

Bujold, N. et Saint-Pierre, H. (1996). Style d'intervention pédagogique, relations affectives enseignants-étudiants et engagement par rapport à la matière. La Revue Canadienne D'enseignement Supérieur, XXVI. Repéré à http://journals.sfu.ca/cjhe/index.php/cjhe/article/view/183233.

Chamberland, G., Lavoie, L. et Marquis, D. (1995). 20 formules pédagogiques. Sainte-Foy : Presses de l'Université du Québec.

Crespo, M. et Houle, R. (1995). La persévérance aux études dans les programmes de premier cycle à l'Université de Montréal. Montréal : Université de Montréal, Vice-décanat aux études supérieures et à la recherche.

Darling-Hammond, L. (2006). Powerful teacher education: lessons from exemplary programs (1 ${ }^{\text {re }}$ éd.). San Francisco, CA : Jossey-Bass.

Duguet, A. (2014). Les pratiques pédagogiques en première année universitaire : description et analyse de leurs implications sur la scolarité des étudiants (Thèse en éducation, Université de Bourgogne, Dijon). Repéré à https://halshs.archives-ouvertes.fr/tel-01096748.

Eccles, J.S. et Wigfield, A. (2002). Motivational beliefs, values, and goals. Annual Review of Psychology, (53), 109-132. Repéré à http://www.rcgd.isr.umich.edu/garp/articles/eccles02c. pdf.

Freeman, S., Eddy, S.L., McDonough, M., Smith, M.K., Okoroafor, N., Jordt, H. et Wenderoth, M.P. (2014). Active learning increases student performance in science, engineering, and mathematics. Proceedings of the National Academy of Sciences of the United States of America, 111(23), 8410-8415. DOI: $10.1073 /$ pnas.1319030111.

Good, T. L. et Brophy, J. E. (2000). Looking in classroom (8 éd). New-York, NY : Longman.

Gouvernement du Québec (2014). Indicateurs de l'éducation. Québec: Ministère de l'Éducation, du Loisir et du Sport. Repéré à http://www.education.gouv.qc.ca/fileadmin/site_web/documents/ PSG/statistiques_info_decisionnelle/Indicateurs_educ_2013_webP.pdf.

Halawah, I. (2011). Factors influencing college students' motivation to learn from students' perspective. Education, 132(2), 379-390. Repéré à http://connection.ebscohost.com/c/casestudies/70532607/factors-influencing-college-students-motivation-learn-from-studentsperspective.

Huang, K.S., Wang, C. et Wang, T.P. (2012). Utilizing Problem-based Learning (PBL) in a University English Interpretation Class. The Journal of Human Resource and Adult Learning, 8(1), 7-15. Repéré à http://www.hraljournal.com/Page/2\%20Tzu-Pu\%20Wang.pdf.

Joyce, B., Weil, M. et Calhoun, E. (2015). Models of Teaching ( $9^{\mathrm{e}}$ éd.). New Jersey : Pearson education. Leclerc, C., Bourassa, B. et Macé, C. (2017). Dérives de la recherche et détresse psychologique chez les universitaires. Perspectives interdisciplinaires sur le travail et la santé, 19(2), 1-48. DOI : 10.4000/ pistes.5155.

Legendre, R. (2005). Dictionnaire actuel de l'éducation (3éd.). Montréal : Guérin.

Loiola, F.-A. et Romainville, M. (2008). La recherche sur la pédagogie de l'enseignement supérieur. Où en sommes-nous ?. Revue Des Sciences de L'éducation, 34(3), 529-535. DOI : 10.7202/029507ar. 
Ménard, L. (2017). Les effets de la formation à l'enseignement et de l'accompagnement des nouveaux professeurs d'université. Dans P. Pelletier et A. Huot (dir.), Construire l'expertise pédagogique et curriculaire en enseignement supérieur : Connaissances, compétences et expériences (p. 33-54). Québec: Presses de l'Université du Québec.

Ménard, L. et Leduc, D. (2016). La motivation à apprendre des étudiants de français et de littérature au cégep. Revue internationale de pédagogie de l'enseignement supérieur, 32(2), 1-14. Repéré à http://ripes.revues.org/1063.

Merriam, S.B. (1988). Case study research in education. San Francisco, CA: Jossey-Bass Publishers.

Messier, G. (2014). Proposition d'un réseau conceptuel initial qui précise et illustre la nature, la structure ainsi que la dynamique des concepts apparentés au terme méthode en pédagogie. (Thèse en éducation, Université du Québec à Montréal, Montréal). Repéré à http://www.archipel.uqam.ca/6822/1/ D2770.pdf.

Pintrich, P.R. et De Groot, E.V. (1990). Motivational and Self-Regulated Learning Components of Classroom Academic Performance. Journal of Educational Psychology, 82(1), 33-40. DOI 2048/10.1037/0022-0663.82.1.33.

Pintrich, P.R. et Schrauben, B. (1992). Students' motivational beliefs and their cognitive engagement in classroom tasks. Dans D. Schunk et J.E. Meece (dir.), Student perceptions in the classroom: Causes and consequences (p. 149-183). Hillsdale, MI: Erlbaum.

Pratt, D.D. (2005). Five perspectives on teaching in adult and higher education (Édition réimprimée). Malabar Krieger Pub. Co.

Prégent, R. (1990). La préparation d'un cours. Montréal : Éditions de l'École Polytechnique de Montréal.

Prégent, R., Bernard, H. et Kozanitis, A. (2009). Enseigner à l'université dans une approcheprogramme : guide à l'intention des nouveaux professeurs et chargés de cours. Montréal : Presses internationales Polytechnique.

Raby, C. et Viola, S. (dir.). (2016). Modèles d'enseignement et théories d'apprentissage : De la théorie à la pratique ( $2^{\mathrm{e}}$ éd.). Anjou : Les éditions CEC.

Sauvé, L., Debeurme, G., Wright, A., Fournier, J. et Fontaine, E. (2007). L'abandon et la persévérance aux études postsecondaires. Rapport d'expérimentation. Rapport final présenté au FQRSC. Actions concertées. Repéré à http://www.savie.qc.ca/CampusVirtuel/Upload/Fichiers/SAMI-

Perseverance_rapport_recension_15-06-09-VF.pdf.

Tinto, V. (2012). Completing college: rethinking institutional action. Londres: The University of Chicago Press Chicago and London.

Tournier, M. (1978). Typologies des formules pédagogiques. Québec : Ministère de l'Éducation, Service général des communications.

Tremblay-Wragg, É. (2013). Conception des étudiants en formation initiale des maîtres du rôle des formateurs universitaires dans leur utilisation d'approches pédagogiques diversifiées en stage (Mémoire en éducation, Université du Québec à Montréal, Montréal). Repéré à http:// www.archipel.uqam.ca/6266/1/M12974.pdf.

Van Der Maren, J.-M. (2003). La recherche appliquée en pédagogie ( $2^{\mathrm{e}}$ éd.). Montréal : Les Presses de l'Université de Montréal, Bruxelles : De Boeck (1e éd. 1996).

Viau, R. (2006). La motivation des étudiants à l'université : mieux comprendre pour mieux agir (p. 1-9). Document inédit présenté dans le cadre d'une conférence, Université de Liège, Belgique. 
Viau, R. (2009). La motivation à apprendre en milieu scolaire. Saint-Laurent : Éditions du Renouveau Pédagogique.

Viau, R. (2014). Savoir motiver les étudiants. Dans L. Ménard et L. St-Pierre (dir.), Se former à la pédagogie de l'enseignement supérieur (p. 235-254). Montréal : Collection Performa.

Viau, R., Joly, J. et Bédard, D. (2004). La motivation des étudiants en formation des maîtres à l'égard d'activités pédagogiques innovatrices. Revue des sciences de l'éducation, 30(1), 163-176. DOI : 10.7202/011775ar.

Vienneau, R. (2017). Apprentissage et enseignement : Théories et pratiques ( $3^{\mathrm{e}}$ éd.). Montréal : Gaëtan Morin éditeur.

\section{NOTES}

1. Cette recherche a reçu l'appui financier du Conseil de Recherche en Sciences Humaines et du Fonds de Recherche du Québec Société et Culture par le biais d'une bourse de doctorat octroyée à la première auteure.

2. Afin de préserver l'anonymat des participants, le nom du professeur utilisé dans cet article est fictif.

\section{RÉSUMÉS}

Plusieurs chercheurs en éducation soutiennent qu'une pluralité de stratégies pédagogiques permet de profiter des avantages de chacune d'entre elles. En effet, selon Sauvé, Debeurme, Wright, Fournier et Fontaine (2007), des activités pédagogiques diversifiées offrent aux étudiants la possibilité de faire des choix et d'enrayer la routine tout en favorisant leur motivation à apprendre. Pourtant, cette pluralité de stratégies pédagogiques utilisée dans le cadre d'un même cours en lien avec la motivation à apprendre des étudiants qui en bénéficient reste peu documentée. En réponse à ce manque, cet article décrit le cas d'un professeur universitaire qui utilise plusieurs stratégies pédagogiques dans son enseignement. Les résultats de cette étude de cas, menée à l'aide d'un entretien avec le professeur, de 12 heures d'observation en classe, d'un questionnaire sur la motivation administré à trois reprises à tous les étudiants du groupe et d'entretiens menés avec dix d'entre eux, démontrent entre autres que l'enseignement dispensé par cet enseignant, en plus d'être diversifié, est motivant pour les étudiants. En effet, Carlos sort du cadre de l'enseignement traditionnel en utilisant 14 stratégies pédagogiques différentes dans son cours, offrant des occasions d'apprentissage variées qui suscitent fortement la motivation de ses étudiants. L'article présente et discute des facteurs qui contribuent à ce niveau élevé de motivation des étudiants en accordant une place importante aux stratégies pédagogiques.

Many educational researchers support a variety of educational strategies to reap the benefits of each. Indeed, according to Sauvé, Debeurme, Wright, Fournier and Fontaine (2007), diversified pedagogical activities offer students the opportunity to make choices and break the routine while encouraging their motivation to learn. However, the motivation to learn from students who are subject to several educational strategies within the same course remains poorly documented. In 
response to this situation, this article describes the case of a university professor who uses several teaching strategies in his teaching. This case study was conducted through an interview with the professor, 12 hours of classroom observation and a questionnaire on motivation completed on three occasions during the session by his students as well as interviews with ten of them. The findings show that the methods employed by this professor, in addition to being diversified, are motivating for the students. Indeed, Carlos steps out of the traditional teaching framework by using 14 different teaching strategies as part of his course, thus providing a variety of learning opportunities that motivate his students. The article presents and discusses the factors contributing to this high level of student motivation by placing a strong emphasis on the instructional strategies.

\section{INDEX}

Mots-clés : pédagogie universitaire, étude de cas, stratégies pédagogiques diversifiées, motivation à apprendre, réussite et persévérance scolaires

\section{AUTEURS}

\section{ÉMILIE TREMBLAY-WRAGG}

Doctorante, UQAM, Montréal

tremblay-wragg.emilie@uqam.ca

\section{CAROLE RABY}

Professeure, UQAM, Montréal

raby.carole@uqam.ca

\section{LOUISE MÉNARD}

Professeure, UQAM, Montréal

menard.louise@uqam.ca 